\title{
Ministry of Health Clinical Practice Guidelines: Dementia
}

\author{
Nagaendran K, Chen LH Christopher, Chong MS, Chua Esther Vanessa, Goh CK Shirley, Kua Joshua, \\ Lee Theresa, Marziyana AR, Ng CC David, Ng LL, Seow Dennis, Sitoh YY, Yap LK Philip, Yeo Donald, Yeo Y
}

\begin{abstract}
The Ministry of Health $(\mathrm{MOH})$ has updated the clinical practice guidelines on Dementia to provide doctors and patients in Singapore with evidence-based treatment for dementia. This article reproduces the introduction and executive summary (with recommendations from the guidelines) from the $\mathrm{MOH}$ clinical practice guidelines on Dementia, for the information of readers of the Singapore Medical Journal. Chapters and page numbers mentioned in the reproduced extract refer to the full text of the guidelines, which are available from the Ministry of Health website: http://www.moh.gov.sg/content/moh_web/healthprofessionalsportal/doctors/guidelines/cpg_medical/2013/ cpgmed_dementia_revised.html. The recommendations should be used with reference to the full text of the guidelines. Following this article are multiple choice questions based on the full text of the guidelines.
\end{abstract}

\section{INTRODUCTION}

\subsection{Objectives and scope of guideline}

The aim of the guidelines is to provide guidance to healthcare professionals in Singapore to assess, evaluate and manage dementia in their patients. This booklet includes chapters on epidemiology, diagnosis, screening and management of dementia. There is discussion on ethical and legal issues related to dementia, palliative care and young-onset dementia. Useful information on community resources are also provided.

\subsection{Target group}

The target group of the guidelines include primary care and specialist doctors, nurses and all allied health staff involved in the care of patients with dementia.

\subsection{Guideline development}

These guidelines have been produced by a committee comprising general practitioners, neurologists, geriatricians, psychiatrists, psychologists, dementia nurses, social workers and patient representatives appointed by the Ministry of Health. They were developed using the best available current evidence and expert opinion.

\subsection{What's new in the revised guidelines}

The following is a list of major revisions or additions to the guidelines:

(1) Updated recommendations on the pharmacological treatment of the different types of dementia.

(2) Discussion on the approach to informing patients and carers on the diagnosis of dementia.
(3) Diagnostic considerations for Mild Cognitive Impairment (MCl).

(4) Ethical and legal issues with discussion of the Mental Capacity Code of Practice 2008.

(5) Section on the management aspects of Young Onset Dementia.

\subsection{Review of guidelines}

Evidence-based clinical practice guidelines are only as current as the evidence that supports them. Users must keep in mind that new evidence could supersede recommendations in these guidelines. The workgroup advises that these guidelines be scheduled for review five years after publication, or if new evidence appears that requires substantive changes to the recommendations.

\section{EXECUTIVE SUMMARY OF RECOMMENDATIONS}

Details of the recommendations listed can be found in the main text as the pages indicated.

\section{Diagnosis and screening}

C Individuals who should be evaluated for dementia include those with progressive cognitive or behavioural complaints suggestive of dementia, as well as patients who arouse the physician's or caregiver's suspicion of cognitive impairment despite the absence of complaints (pg 17).

Grade C, Level 2+

List of institutions in alphabetical order

Age-Link Specialist Clinic for Older Persons (Mt Elizabeth), Alzheimer's Disease Association, Institute of Mental Health, Khoo Teck Puat Hospital, Ministry of Health, National Healthcare Group Polyclinics - Chua Chu Kang, National Neuroscience Institute (Tan Tock Seng Hospital Campus), National University of Singapore, Singapore General Hospital, Singhealth Polyclinics - Queenstown, St Luke's Hospital, Tan Tock Seng Hospital

Correspondence: Dr Nagaendran Kandiah, Consultant, Department of Neurology, National Neuroscience Institute, Tan Tock Seng Hospital, 11 Jalan Tan Tock Seng, Singapore 308433. Nagaendran_Kandiah@nni.com.sg 
GPP Assessment of dementia should be done via a comprehensive evaluation. This approach will aim to diagnose dementia early, assess for complications of dementia and establish the cause of dementia (pg 18).

B In individuals with suspected cognitive impairment, diagnosis should be made using the DSM-IV criteria for dementia with history from a reliable informant. This should be supplemented by an objective approach, with bedside cognitive tests and/or neuropsychological assessment (pg 18).

Grade B, Level 2++

D In the evaluation of suspected dementia, the presence of depression should be considered (pg 21).

Grade D, Level 4

B The complications of dementia can be broadly divided into behavioural and psychological symptoms, functional problems and social problems. These should be evaluated in all patients with dementia, as these issues are the major causes of stress on the caregiver and assessment would enable the clinician to effectively target subsequent management (pg 22).

Grade B, Level 2++

In the clinic setting, the physician should routinely ask about depression, anxiety, agitation, paranoia, hallucinations and sleep problems, as these are potentially amenable to treatment, by way of either psychosocial interventions or pharmacologic agents (pg 22).

D The aim of determining dementia aetiology is to rule out potentially reversible causes of dementia and selecting appropriate treatment strategies for the irreversible dementias. This should be done via clinical history and physical examination, followed by laboratory investigations and neuroimaging where appropriate (pg 24).

Grade D, Level 4

Screening for neurosyphilis should be considered for patients with young onset dementia, patients with a history of sexually transmitted diseases and patients presenting with a neuropsychiatric syndrome (pg 25).

B Clinicians should make a diagnosis of a specific type of dementia based on the available criteria. A number of well-validated clinical criteria may be used for the various types of dementia (Alzheimer's disease, vascular dementia, dementia with Lewy bodies, Parkinson's disease dementia and fronto-temporal dementia) (pg 26).

Grade B, Level 2++

D Patients' and, where appropriate, their family's preferences for disclosure should be sought with respect to the diagnosis of dementia, and acted upon accordingly. The communication of diagnoses should be done in a sensitive and empathic manner, and the patient and family should be given time to come to terms with the diagnosis (pg 27).

Grade D, Level 4

GPP Genetic testing should not be routinely carried out in the clinical evaluation of dementia (pg 29).

GPP

B Routine testing of the Apolipoprotein E (APOE) gene is not recommended in dementia diagnosis and in tailoring dementia risk reduction (pg 30).

Grade B, Level 2++

\section{Pharmacological management of dementia}

GPP Pharmacotherapy should be part of a multi-pronged strategy to dementia management, which encompasses a well-established diagnosis and consideration of stage-specific challenges such as education of patient and caregiver, non-pharmacological measures and comprehensive caregiver psychosocial intervention (pg 31).

GPP

Pharmacotherapy should not be used in isolation in the management of dementia, but in conjunction with nonpharmacological management, including education and counselling of the patient and caregiver (pg 31).

GPP

Patients with dementia should be screened and treated for reversible identifiable causes (such as depression, B12 deficiency and hypothyroidism) and vascular risk factors (pg 32).

GPP

GPP The current evidence for the use of cognitive enhancers is generally based on clinical trials of up to one year's duration. The use of cognitive enhancers for longer periods will need to include a detailed discussion with the patient and caregiver on the overall benefit of treatment and specific needs of the patient (pg 34).

GPP

A Acetylcholinesterase inhibitors (donepezil, galantamine or rivastigmine) should be considered for the management 
of patients with mild to moderate Alzheimer's disease (pg 34).

$$
\text { Grade A, Level 1++ }
$$

A Acetylcholinesterase inhibitors may be considered for the management of moderately severe to severe Alzheimer's disease (pg 35).

Grade A, Level 1+

A Where tolerated, acetylcholinesterase inhibitors should be titrated to the recommended doses (5-10 mg/day donepezil; 16-24 mg/day galantamine; 6-12 mg/day oral and $4.6-9.5 \mathrm{mg} / 24 \mathrm{hr}$ transdermal rivastigmine), which have been shown to confer greater benefit compared with lower doses (pg 35).

Grade A, Level 1++

A N-methyl D-aspartate antagonists (memantine) may be considered for the management of moderately severe to severe Alzheimer's disease, either alone or in combination with acetylcholinesterase inhibitors (pg 36).

Grade A, Level 1+

A N-methyl D-aspartate antagonists (memantine) may be considered for the treatment of mild to moderate Alzheimer's disease, if acetylcholinesterase inhibitor therapy is contraindicated, not tolerated, or if there is disease progression despite an adequate trial of acetylcholinesterase inhibitors (pg 36).

Grade A, Level 1+

A Acetylcholinesterase inhibitors have been shown to be of clinical benefit, and may be considered for use in the management of mild to moderate vascular dementia (pg 37).

Grade A, Level 1+

A N-methyl D-aspartate antagonists (memantine) have been shown to be of clinical benefit, and may be considered for use in the management of mild to moderate vascular dementia (pg 38).

Grade A, Level 1+

GPP Appropriate treatment of vascular risk factors and lifestyle changes, including a healthy diet and regular exercise, is recommended for all patients with vascular dementia (pg 39).

GPP

B Acetylcholinesterase inhibitors may be considered for the management of cognitive and behavioural symptoms related to Parkinson's disease dementia (pg 39).

Grade B, Level 1+
B Acetylcholinesterase inhibitors or $\mathrm{N}$-methyl D-aspartate antagonists (memantine) may be considered for the management of cognitive and behavioural symptoms related to dementia with Lewy bodies (pg 40).

Grade B, Level 1+

B Selective serotonin reuptake inhibitors (SSRIs) may be considered for the management of behavioural symptoms related to frontotemporal dementia (pg 40).

Grade B, Level 1+

C N-methyl D-aspartate antagonists (memantine) and acetylcholinesterase inhibitors may be considered in the management of cognitive and behavioural symptoms related to frontotemporal dementia (pg 40).

Grade C, Level 2+

GPP The choice of acetylcholinesterase inhibitors should be based upon factors such as the experience of the clinician, tolerance to side effects, ease of use, and the clinical profile of the individual to be treated (pg 41).

GPP

GPP The decision to initiate cognitive enhancers such as acetylcholinesterase inhibitors or $\mathrm{N}$-methyl D-aspartate antagonists (memantine), should be made in consultation with the patient and family after careful consideration of the expected magnitude of benefit, side effects, comorbidities and costs of treatment (pg 42).

GPP

B Patients who are started on acetylcholinesterase inhibitors should be monitored for side effects such as nausea, vomiting, diarrhoea and anorexia, and bradycardia (pg 43).

Grade B, Level 1+

GPP Patients who are started on cognitive enhancers should be assessed for cognition, mood and behaviour, and function within 3-6 months of starting therapy and thereafter, at least once yearly or as clinically indicated (pg 43).

GPP

A Anti-inflammatory agents (such as nonsteroidal antiinflammatory agents and cyclo-oxygenase 2 inhibitors) are not recommended for the prevention of cognitive decline in Alzheimer's disease (pg 44).

Grade A, Level 1++

B Prednisolone is not recommended for the prevention of cognitive decline in Alzheimer's disease (pg 44).

Grade B, Level 1+ 
A Oestrogen is not recommended for the prevention of cognitive decline in women with Alzheimer's disease (pg 44).

Grade A, Level 1++

A Selegiline is not recommended for the treatment of core or associated symptoms in Alzheimer's disease (pg 44).

Grade A, Level 1+

B High dose vitamin E (in excess of $400 \mathrm{IU}$ per day) is not recommended for the prevention or treatment of Alzheimer's disease (pg 45).

Grade B, Level 1+

B Ginkgo is not recommended for the routine treatment of dementia (pg 45).

Grade B, Level 1+

A Omega 3 fatty acid is not recommended for the prevention or routine treatment of dementia (pg 45).

Grade A, Level 1+

A Statin therapy is not recommended for the prevention or routine treatment of Alzheimer's disease (pg 46).

Grade A, Level 1++

A Folic acid and vitamin B supplementation are not recommended for the prevention and treatment of dementia in the absence of B vitamin deficiency (pg 46).

Grade A, Level 1++

A Rosiglitazone is not recommended as monotherapy or as adjunctive therapy to cholinesterase inhibitors in mild to moderate Alzheimer's disease (pg 46).

Grade A, Level 1+

A Multi-component and individualised caregiver interventions should be considered for holistic dementia care (pg 49).

Grade A, Level 1+

GPP Where appropriate, respite care may be offered to relieve the burden of caregiving on caregivers of persons with dementia (pg 50).

B Therapy incorporating cognitive and behavioural strategies may be considered for persons with dementia (pg 51).

Grade B, Level 1+

\section{Management of behavioural and psychological} symptoms of dementia

C Environmental design features may be incorporated in care facilities to reduce behavioural and psychological symptoms of dementia (BPSD) in people with dementia (pg 52).

Grade C, Level 2+

D Art therapy may be considered for persons with behavioural and psychological symptoms of dementia (BPSD) (pg 52).

Grade D, Level 3

B Reminiscence therapy (RT) may be considered for persons with behavioural and psychological symptoms of dementia (BPSD) (pg 52).

Grade B, Level 1+

C Persons with dementia may be encouraged to participate in structured exercise programmes to improve physical function (pg 53).

Grade C, Level 2+

B Music therapy, wherever feasible, is encouraged in the care of persons with dementia, and is helpful in ameliorating the behavioural and neuropsychiatric symptoms of dementia (pg 53).

Grade B, Level 1+

B Aromatherapy is not recommended for reducing agitation in persons with Alzheimer's disease (pg 55).

Grade B, Level 1+

B Massage therapy could be considered in reducing agitation in persons with Alzheimer's disease (pg 56).

Grade B, Level 1+

A Multisensory stimulation is not recommended in the care of elderly patients with dementia (pg 56).

Grade A, Level 1+

GPP Antidepressants may be used for the treatment of comorbid depression in dementia, provided their use has been evaluated carefully for each patient (pg 57).

GPP

A Antipsychotic medications may be considered in the treatment of behavioural and psychological symptoms of dementia when clinically appropriate and nonpharmacological management has not been useful (pg 57).

Grade A, Level 1++ 
B Potential side-effects and risk/benefit ratio of antipsychotic medication should be discussed with patients and/or caregivers (pg 58).

Grade B, Level 2+

A Routine use of mood stabilisers, such as carbamazepine and sodium valproate, is not recommended for the treatment of behavioural symptoms associated with dementia (pg 59).

Grade A, Level 1+

GPP An individualised approach to managing behavioural problems in dementia patients is required (pg 59).

GPP

GPP Acetylcholinesterase inhibitor therapy may be considered in the treatment of patients with behavioural problems if antipsychotics are inappropriate (pg 59).

GPP

GPP The decision to start antipsychotic therapy to control behavioural problems in dementia patients should be made in consultation with the patient and family, after careful consideration of the benefit, adverse effects and comorbidities (pg 59).

B For patients with dementia with Lewy bodies and behavioural problems, acetylcholinesterase inhibitors should first be considered for the management of the behavioural problems (pg 60).

Grade B, Level 1+

GPP In all patients started on antipsychotic medication, they should be monitored carefully for side effects and response to treatment. In patients who are stable, antipsychotic withdrawal should be considered (pg 60).

\section{Ethical and legal issues}

GPP Persons with dementia who are deemed to have decisionmaking capacity (after clinical evaluation) are encouraged to make a Lasting Power of Attorney (LPA) (pg 62).

B Routine testing of APOE (Apolipoprotein E) gene is not recommended in dementia diagnosis and in tailoring dementia risk reduction (pg 66).

\section{Grade B, Level 2++}

D The judgement, with respect to the ability to drive safely, of a person with dementia should only be made after a systematic and comprehensive assessment (pg 68).

Grade D, Level 4

D In general, persons whom the physician is unsure if the diagnosis of cognitive impairment might affect driving safety should be referred for further clinical and driving assessment (pg 69).

Grade D, Level 4

GPP In general, the diagnosis of dementia should be disclosed to the patient, unless explicitly stated otherwise (pg 71).

GPP

GPP In disclosure, the doctor should also be mindful of the impact the diagnosis can have on the patient's life and family relationships (pg 71).

GPP

\section{Palliative care}

B Patients with advanced dementia should be assessed for pain and treated accordingly (pg 75).

Grade B, Level 1+

D A stepped protocol is recommended for pharmacological management of pain in dementia, and the WHO analgesic ladder or the American Geriatric Society's pain guidelines may be used (pg 75).

Grade D, Level 4

C Decisions on the use of antibiotics in advanced dementia should be individualised to the patient by weighing the risk and benefits of antibiotic treatment (pg 76).

Grade C, Level 2+

GPP Decisions on tube feeding should be individualised, given the lack of evidence for its efficacy in advanced dementia (pg 77).

GPP

D Advance care planning with regard to cardiopulmonary resuscitation (CPR) should be encouraged, given the poor outcomes of CPR in advanced dementia (pg 78).

Grade D, Level 2+

\section{Young onset dementia}

GPP Patients with young onset dementia should receive specialist multidisciplinary care for the diagnosis and management of their condition (pg 79).

GPP 
GPP In the diagnostic workup of patients with young onset dementia, neuroimaging and cerebrospinal fluid examination should be considered (pg 79).

GPP

GPP Caregivers of patients with young onset dementia should receive adequate counselling on the disease process, community resources and financial assistance (pg 79).

GPP

\section{Community resources}

B Caregivers of persons with advanced dementia should be provided with adequate information on the available community and medical resources (pg 80).

Grade B, Level 2

B Referral to day care services and respite services should be considered as part of a comprehensive management plan (pg 81).

Grade B, Level 2 


\section{SINGAPORE MEDICAL COUNCIL CATEGORY 3B CME PROGRAMME (Code SMJ 201305C)}

These questions are based on the full text of the guidelines, which may be found at http://www.moh.gov.sg/content/moh_web/ healthprofessionalsportal/doctors/guidelines/cpg_medical/2013/cpgmed_dementia_revised.html.

Question 1. With regard to the diagnosis of dementia:

(a) Dementia can be diagnosed clinically based on the DSM IV criteria.

(b) Neuropsychological evaluation is a requirement for the diagnosis of dementia.

(c) Corroborative history from a reliable caregiver/informant improves the diagnostic certainty of dementia.

(d) Neuroimaging should always be performed prior to making a diagnosis of dementia.

Question 2. In the pharmacological management of dementia with cognitive enhancers:

(a) Acetylcholinesterase inhibitors may be used for mild, moderate and severe stages of Alzheimer's dementia.

(b) N-methyl D-aspartate antagonists (memantine) may be used for the treatment of mild Alzheimer's dementia if the patient has contraindications to acetylcholinesterase inhibitors.

(c) Combination of acetylcholinesterase inhibitors and $\mathrm{N}$-methyl $\mathrm{D}$-aspartate antagonists (memantine) may be considered in selected patients.

(d) Where tolerated, dosages should be titrated to the recommended doses.

Question 3. When providing education to patients and caregivers:

(a) The cost-benefit ratio of treatment with cognitive enhancers should not be discussed.

(b) It is important to discuss the prognosis and potential complications of dementia.

(c) Discussion on social and community resources should be included.

(d) The importance of family support should be incorporated.

Question 4. In the evaluation of dementia:

(a) Neuroimaging can increase the diagnostic accuracy and identify potential reversible causes.

(b) Genetic testing is recommended for all patients with dementia.

(c) Testing for neurosyphilis may be required for selected high-risk patients.

(d) For patients with young onset dementia, evaluation for infective, inflammatory and neoplastic aetiology should be considered.

Question 5. In patients with mild dementia:

(a) A person must be assumed to have capacity unless it is established that he lacks capacity.

(b) Patients deemed to have intact judgement should be encouraged to make a Lasting Power of Attorney.

(c) Patients who are still driving should be referred for a formal driving assessment.

(d) Treatment with ginkgo has been demonstrated to improve cognitive function.

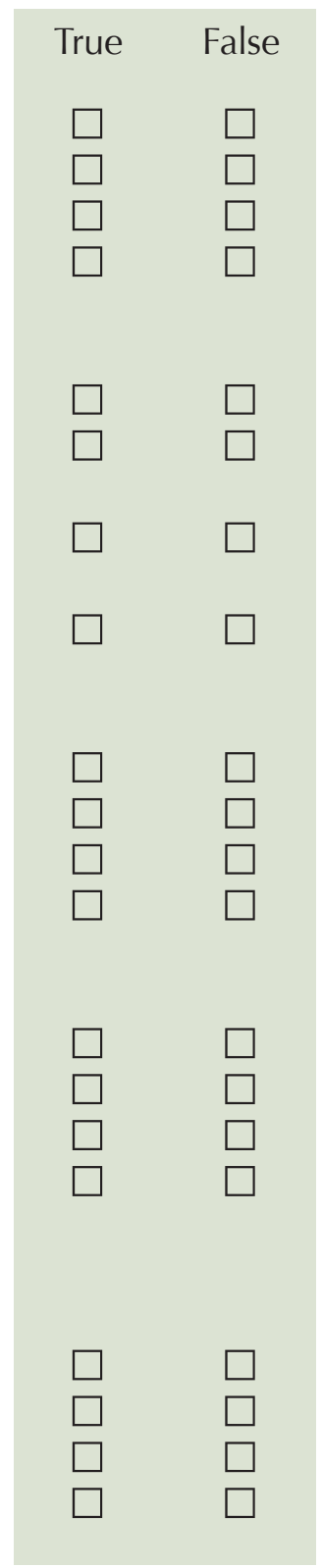

\footnotetext{
Doctor's particulars:

Name in full

MCR number

Email address

SUBMISSION INSTRUCTIONS:

(1) Log on at the SMJ website: http://www.sma.org.sg/publications/smjcurrentissue.aspx and select the appropriate set of questions. (2) Provide your name, email address and MCR number. (3) Select your answers and click "Submit".

RESULTS:

(1) Answers will be published in the SMJ July 2013 issue. (2) The MCR numbers of successful candidates will be posted online at the SMJ website by 27 June 2013. (3) Passing mark is $60 \%$. No mark will be deducted for incorrect answers. (4) The SMJ editorial office will submit the list of successful candidates to the Singapore Medical Council. (5) One CME point is awarded for successful candidates.

Deadline for submission: (May 2013 SMJ 3B CME programme): 12 noon, 20 June 2013.
} 